\title{
Tratamiento y cambio terapéutico en agresores sexuales
}

\author{
Santiago Redondo Illescas ${ }^{1}$ y Ana Martínez Catena \\ Universidad de Barcelona.
}

Manuscrito recibido el 14 de diciembre de 2011 / Publicado el 28 de febrero de 2012

\section{RESUMEN}

Este estudio analiza la relación entre participación de los agresores sexuales en un tratamiento y su disposición terapéutica. La perspectiva utilizada para ello es el modelo transteórico de Prochaska y DiClemente, que estructura la disposición al cambio de conducta en cuatro estadios: precontemplación, contemplación, acción y mantenimiento. La muestra evaluada se dividió en tres grupos: grupo pre-tratamiento, integrado por 67 agresores no tratados; grupo tratamiento, formado por 33 delincuentes sexuales que recibían tratamiento; y grupo seguimiento, compuesto por 19 agresores sexuales que ya habían finalizado el tratamiento. La evaluación de la disposición para el cambio de conducta se realizó mediante la Escala de Estadios de Cambio (SOCS). En consonancia con el modelo transteórico, los resultados evidenciaron una relativa correspondencia entre los diferentes momentos del tratamiento y los sucesivos estadios de cambio terapéutico. Se comenta la complejidad de este campo de análisis y las dificultades existentes para obtener resultado nítidos en la asociación tratamiento-cambio terapéutico.

Palabras clave: agresores sexuales, motivación de cambio, desistencia delictiva, modelo transteórico

\footnotetext{
${ }^{1}$ Correspondencia a autor: Sredondo@ub.edu
} 


\begin{abstract}
This study examines the relationship between participation of sex offenders in a treatment program and their therapeutic readiness. The perspective used for this is the transtheoretical model of Prochaska and DiClemente, which structures the willingness to change behavior in four stages: precontemplation, contemplation, action and maintenance. The evaluated sample was divided into three groups: pre-treatment, consisting of 67 untreated offenders; treatment group, made up of 33 offenders who received treatment; and follow-up group, composed of 19 sex offenders who had completed treatment. The assessment of readiness for behavior change was performed by the Stages of Change Scale (SOCS). Consistent with the transtheoretical model, the results showed a relative correspondence between the different stages of treatment and the successive stages of therapeutic change. The complexity of this field of analysis and the difficulties of obtaining clear results in the association-treatment therapeutic change are commented.
\end{abstract}

Keywords: sex offenders, motivation to change, crime desistance, transtheoretical model

\title{
1. INTRODUCCIÓN
}

\subsection{Desistencia delictiva: necesidad de cambios internos y externos}

La desistencia del delito, o finalización por los delincuentes de su actividad criminal previa, constituye uno de los procesos más relevantes, y también más desconocidos, a que puede dirigirse el análisis criminológico. Se sabe poco acerca de si los delincuentes experimentan un insight repentino hacia el desistimiento o, más bien, se trata de un proceso gradual (Serin y Lloyd, 2009). Diversas investigaciones han analizado la influencia descriminalizadora que tendrían tanto las nuevas experiencias prosociales habidas por los sujetos (familiares, laborales, etc.) como los cambios operados en sus estados subjetivos (Aresti, Eatough y Brooks-Gordon, 2010). En relación con eventuales vivencias prosociales, ha mostrado ser un firme factor protector del delito el adquirir nuevas relaciones interpersonales tales como vinculación de pareja u otras. Por ejemplo, McGloin, Sullivan, Piquero, Blokland, y Nieuwbeerta (2011) hallaron en una muestra de 4000 delincuentes evaluados en Holanda que contraer matrimonio se asociaba a la reducción de su versatilidad delictiva, probablemente debido al cambio de sus 
rutinas vitales y a una menor exposición a oportunidades delictivas. En lo tocante a los cambios internos, Aresti et al. (2010) concluyeron que el factor clave de la desistencia delictiva era la evolución de los sujetos hacia una nueva identidad prosocial. Siendo la mayor barrera para el abandono delictivo el choque entre esta identidad renovada y el encasillamiento social del sujeto como "exdelincuente".

Con todo, lo más probable es que el proceso de desistencia delictiva requiera tanto cambios externos al sujeto como transformaciones internas, tales como la mejora de su autoconcepto y su percepción de autoeficacia (Laub, Nagin y Sampson, 1998). Como ejemplo de la necesidad de ambos factores, LeBel, Burnett, Maruna, y Bushway (2008) hallaron, en un estudio de entrevista con 130 delincuentes, que el desistimiento del delito fue favorecido de forma combinada: mientras que los elementos subjetivos, como sentirse a disgusto con el propio pasado delictivo y creer en la capacidad personal para "salir adelante", fueron una precondición para el abandono de la delincuencia, resultó a la vez necesario que el sujeto contara con oportunidades prácticas para desarrollar una vida prosocial.

Los cambios internos que son imprescindibles para la desistencia delictiva, que constituyen el objetivo científico de este trabajo, pueden producirse de modo natural, como resultado de la maduración del individuo, o ser impulsados mediante la aplicación de un tratamiento. El tratamiento de los delincuentes suele orientarse a favorecer el denominado cambio terapéutico, que incluye mejoras cognitivas, emocionales, actitudinales y de conducta que se asocian a la disminución del riesgo delictivo (Redondo, 2008). Para que el cambio terapéutico se produzca acostumbra a considerarse necesario que el participante en un tratamiento esté motivado para mejorar su conducta (Barbaree y Marshall, 2006; Brown, 2005; Harkins y Beech, 2007; Hollin y Palmer, 2006; Tierney y McCabe, 2002). Miller (1985) definió la motivación para el cambio del comportamiento como la probabilidad que tiene una persona de iniciar, continuar y mantener un proceso de modificación de su conducta.

Existen varias teorías acerca de la motivación y el cambio terapéuticos. Según el modelo de creencias sobre la salud (MCS), el cambio terapéutico se sustentaría en la creencia de un individuo de ser vulnerable, de tener un problema que resolver, y de que ello le reportará más beneficios personales que costes (Becker, 1974; Glanz, Rimer y Viswanath, 2008; Rosenstock, 1974). La teoría de la motivación 
hacia la protección, sugiere que el cambio es facilitado cuando el individuo percibe su problema como una amenaza y se considera capaz de afrontarlo (Orbell et al., 2009; Plotnikoff et al., 2010; Rogers, 1975). La teoría de la autoeficacia propone que aquellas personas que cuentan con mayor capacidad para explorarse a sí mismas (evaluando sus creencias, emociones, expectativas, etc.) pueden modificar más fácilmente sus formas de pensar y de actuar (Bandura, 1977; Schunk y Pajares, 2009). Por último, la teoría de la acción razonada (TRA) interpreta que el cambio de comportamiento es facilitado tanto por la valoración individual de la propia conducta como por las opiniones que de ella tienen las otras personas (Ajzen y Fishbein, 1980; Mullan y Westwood, 2010; Natan, Beyil y Neta, 2009).

\subsection{El modelo transteórico de cambio de la conducta}

Por encima de las anteriores, la teoría más conocida sobre motivación y cambio terapéutico es el modelo transteórico de Prochaska y DiClemente, formulado a partir de 1982 (Casey, Day y Howells, 2005). Integra tres dimensiones interrelacionadas (Labrador, Echeburúa y Becona, 2000; Prochaska y Norcross, 2001): estadios de cambio, procesos de cambio y niveles de cambio. Los estadios de cambio, identificados en origen mediante análisis empíricos (DiClemente y Prochaska, 1982; Prochaska, Velicer, DiClemente, y Fava, 1988), representan una dimensión temporal, concibiéndose como una progresión de la conducta individual a lo largo del tiempo (Prochaska y Norcross, 2001). Los procesos de cambio se refieren a los factores que producen las mejoras terapéuticas, tales como las variaciones en la concienciación del individuo, su capacidad de auto-reevaluación, de manejo de contingencias y de control de estímulos, o el mejoramiento de sus relaciones de ayuda (Prochaska, DiClemente y Norcross, 1992). El modelo transteórico propone que distintos procesos de cambio (y en consecuencia, distintas técnicas psicológicas susceptibles de impulsarlos) pueden resultar más eficaces en ciertos estadios de cambio que en otros (Prochaska y Norcross, 2001; Rosen, 2000). Por último, los niveles de cambio harían referencia a aquellos aspectos conductuales, cognitivos y emocionales implicados en cualquier disfunción del comportamiento, considerando que existirían cinco niveles de cambio progresivos (Prochaska y DiClemente, 2005): cambio situacional, cognitivo, interpersonal, en el sistema familiar, y en los conflictos interpersonales. 
La estructura más difundida y analizada del modelo transteórico es la correspondiente a los estadios, o momentos temporales, de cambio terapéutico. En la categorización más exhaustiva se han descrito seis estadios de cambio (Labrador, et al., 2000; Prochaska y Norcross, 2001; Redondo, 2008): 1) precontemplación, en que el sujeto aún no se ha hecho consciente de tener un problema que deba resolver y por ello todavía no muestra ninguna intención de cambiar; 2) contemplación, cuando el individuo empieza a adquirir conciencia de las dificultades que tiene y comienza a pensar seriamente en hacer algo al respecto; 3) preparación para la acción, momento en el que el sujeto ha decidido emprender acciones para resolver su problema con cierta inmediatez (suele estimarse como tal el plazo de un mes); 4) acción, fase en la que está dando pasos claramente dirigidos a solucionar sus dificultades (variando para ello sus conductas, experiencias o ambientes) y ya ha realizado algunos progresos; 5) mantenimiento, cuando el individuo ya ha conseguido cambiar su conducta problemática (durante un periodo mínimo de seis meses) y está utilizando estrategias concretas para evitar una recaída; y 6) finalización, momento en el que el sujeto ha podido estabilizar sus logros y dar por resuelto su problema. En una versión simplificada del modelo transteórico los estadios de cambio se redujeron de los seis precedentes a cuatro de ellos (Littrell y Girvin, 2002): 1) precontemplación, 2) contemplación, 3) acción, y 4) mantenimiento.

Esta propuesta de cuatro estadios ha sido evaluada mediante la Escala de Estadios de Cambio (SOCS) (McConnaughy, Prochaska y Velicer, 1983). Hemphill y Howell (2000) hallaron alta validez convergente y discriminante de estos cuatro estadios, a partir de su comparación con las escalas Paulhus Deception Scales (PDS) (Paulhus y Lim, 1994; Paulhus, Trapnell y Chen, 1999) y el Multidimensional Anger Inventory (MAI) (Siegel, 1986). El modelo transteórico se ha utilizado, empleando la escala SOCS y otras escalas análogas, para evaluar la disposición hacia el cambio terapéutico de pacientes con problemas de abuso de alcohol y otras sustancias tóxicas, estrés post-traumático, trastornos alimentarios, ansiedad, depresión, y otros (Norcross, Krebs y Prochaska, 2011).

En lo que aquí nos concierne, el modelo transteórico se ha utilizado también para la evaluación del tratamiento de distintas problemáticas y categorías de delincuentes: conductores infractores (Nochajski y Stasiewicz, 2005; Wells-Parker, 
Kenne, Spratke, y Williams, 2000), delincuentes encarcelados (McMurran, Theodosi y Sellen, 2006), delincuentes juveniles (Hemphill y Howell, 2000), control de la ira (Williamson, Day, Howells, Bubner, y Jauncey, 2003), maltratadores de sus parejas (Alexander y Morris, 2008; Norcross, et al., 2011; Scott y Wolfe, 2003), y agresores sexuales (Polaschek, Anstiss y Wilson, 2010; Tierney y McCabe, 2002, 2004, 2005). La conclusión más general es que el modelo transteórico, y sus correspondientes escalas de evaluación, pueden ser útiles para valorar la disposición hacia el cambio de conducta en delincuentes participantes en un tratamiento (Tierney y McCabe, 2004). Contrariamente a ello, algunos trabajos más críticos consideran que el modelo transteórico es una estructura demasiado global para analizar los matices implicados en el proceso de cambio terapéutico y desistencia delictiva (Burrowes y Needs, 2009; Casey, et al., 2005).

\subsection{Tratamiento y motivación terapéutica}

Se ha debatido si es imprescindible que los delincuentes, antes de participar en un tratamiento, cuenten con una motivación "genuina" y un deseo sincero de cambio, en torno a lo cual se han realizado algunas investigaciones, que han obtenido resultados dispares (Tierney y McCabe, 2002). Algunos estudios han concluido, en la dirección más exigente, que se debería aplicar una intervención terapéutica exclusivamente a aquellos sujetos que están motivados para participar en ella. Sólo un deseo genuino del individuo de abandonar la delincuencia puede facilitar que se beneficie del tratamiento (Harkins y Beech, 2007). Como aval de esta postura, diversos estudios han hallado una correlación positiva entre niveles más elevados de motivación terapéutica y mayor eficacia de la intervención (August y Flynn, 2007; Bowen y Gilchrist, 2004; Pelissier, 2007; Pereira de Oliveira y Malbieger, 2003; Tierney y McCabe, 2002).

Sin embargo, es sumamente difícil que los delincuentes presenten una motivación de cambio plenamente sincera, ya que los tratamientos suelen desarrollarse en un contexto de privación de libertad. Los motivos que los delincuentes encarcelados pueden tener para participar en una intervención son variados, no exclusivamente terapéuticos. Por ello, desde una perspectiva más posibilista, se ha considerado que es el tratamiento el que debe promover o desarrollar progresivamente en los sujetos su motivación terapéutica, sin que ésta 
deba considerarse una precondición (Day, Gerace, Wilson, y Howells, 2008; Grubin y Thornton, 1994; Tierney y McCabe, 2002, 2004). En un programa dirigido a erradicar las distorsiones cognitivas de dos categorías de agresores sexuales, Terry y Mitchel (2001) obtuvieron resultados ambivalentes acerca del influjo de la motivación previa en el éxito del tratamiento. Para el caso de los agresores sexuales de mujeres adultas, el tratamiento produjo efectos positivos similares en los participantes que contaban con precedente motivación de cambio y en los que carecían de ella. Por el contrario, la motivación previa para cambiar potenció la eficacia del tratamiento en los abusadores de menores.

Un planteamiento de compromiso entre los dos anteriores (máxima exigencia motivacional para el tratamiento vs mínima) afirmaría la necesidad de aplicar tratamiento incluso a sujetos no altamente motivados para participar en el mismo, pero adaptando los objetivos de la intervención al nivel motivacional que presentan (Day, Bryan, Davey y Casey, 2006; DiClemente, Garay y Gemmell, 2008; Eckhardt, Babcock y Homack, 2004; Harkins y Beech, 2007; Prochaska y Norcross, 2001; Tierney y McCabe, 2004).

Se ha considerado que los agresores sexuales muestran en general escasa motivación para cambiar debido a la frecuente negación, minimización y justificación que realizan de sus delitos (Garland y Dougher, 1991; Harkins y Beech, 2007; Kear-Colwell y Pollock, 1997; Tierney y McCabe, 2002). Por ello, para una mayor eficacia terapéutica, se estima muy conveniente que los delincuentes sexuales reconozcan los delitos cometidos, abandonen sus habituales procesos de justificación, y se responsabilicen de los daños causados a otras personas (Barrett, Wilson y Long, 2003; Harkins y Beech, 2007; Redondo, 2006). Para favorecer estas mejoras, Marshall y Moulden (2006) han propuesto aplicar pre-programas motivacionales que reduzcan la resistencia inicial de los agresores sexuales hacia el reconocimiento delictivo y el abandono del delito. Pese a todo, Marshall y Pithers (1994) interpretaron que muchos delincuentes sexuales, más que presentar una resistencia general a variar su conducta, lo que en realidad muestran es una dificultad para modificarla de forma rápida. Es decir, lo que sucede es que distintos agresores sexuales pueden cambiar a diferentes ritmos, dependiendo de aspectos como la gravedad de sus delitos, las habilidades y recursos personales con los que cuentan, y las diferencias en su motivación. 
Como puede verse, por todo lo comentado hasta aquí, el análisis de las relaciones entre el tratamiento y la motivación y el cambio terapéutico de los agresores sexuales es un ámbito de investigación complejo y con resultados ambivalentes. Aun así, el modelo transteórico de Prochaska y DiClemente constituye la conceptualización más sólida e internacionalmente utilizada para adaptar las intervenciones con los delincuentes al momento motivacional de cada participante (Prochaska y Norcross, 2001), algo sobre lo que no existen estudios en España. El modelo transteórico establece que, cuando las personas varían su conducta, transitan por diversos estadios de cambio (precontemplación, contemplación, acción y mantenimiento). Y un presupuesto importante, deducible de una parte de la investigación en este campo, es que la disposición a la modificación de la propia conducta, y en consecuencia el avance en estadio de cambio, pueden ser favorecidos mediante el tratamiento (Day, et al., 2008; Grubin y Thornton, 1994; Tierney y McCabe, 2002, 2004). Bajo estas premisas sería razonable esperar que los agresores sexuales que no han comenzado un programa, o se hallan en sus primeras fases, se situaran en estadios de cambio iniciales. Y que aquellos otros que llevan tiempo participando en un tratamiento, o ya lo han finalizado, se ubicaran en estadios más avanzados. La correspondencia ideal entre fases sucesivas del tratamiento y estadios de cambio en que se sitúan los participantes podría constituir un criterio teórico y empírico razonable del buen funcionamiento de un programa y de su eventual eficacia terapéutica.

En consonancia con lo anterior, el propósito de esta investigación es explorar la utilidad del modelo transteórico (Prochaska y DiClemente, 1982) en una muestra de agresores sexuales encarcelados. Su objetivo específico es evaluar la relación que pueda existir entre los sucesivos momentos del tratamiento en que se encuentran los sujetos (con antelación a haberlo iniciado, en proceso de tratamiento, o habiéndolo ya finalizado) y sus puntuaciones en los estadios de cambio del modelo transteórico. La hipótesis general es que debería existir reciprocidad entre la fase de tratamiento en que un grupo se halla y su nivel de cambio terapéutico: los sujetos en pre-tratamiento deberían situarse en estadios de cambio iniciales (precontemplación), los del grupo tratamiento en estadios intermedios (contemplación y acción), y los del grupo seguimiento en estadios finales (mantenimiento). 


\section{MÉTODO}

\subsection{Participantes}

La muestra de esta investigación está integrada por 76 sujetos internos en el Centro Penitenciario Brians II (en la provincia de Barcelona), de edades comprendidas en el rango 21-68 años $(M=39,77 ; D S=10,63)$. La muestra global se divide en tres grupos de estudio: a) grupo pre-tratamiento, compuesto por 24 delincuentes que no habían participado todavía en ninguna intervención; b) grupo tratamiento, integrado por 33 internos que cumplían condena por un delito sexual y se hallaban en proceso de tratamiento; y c) grupo seguimiento, formado por 19 agresores sexuales que ya habían finalizado el tratamiento.

Los tres grupos constituidos son homogéneos en sus características sociodemográficas y de carrera criminal, siendo las principales, en las que los grupos presentaban proporciones semejantes, las siguientes: respecto de la nacionalidad, el 55\% de la muestra era de origen español mientras que un 15,5\% procedía de otros países europeos, un 20\% de estados iberoamericanos, y un 9\% de países africanos; la mayoría de los sujetos había cursado estudios básicos (90\%); sólo el 20\% de la muestra mantenía una relación de pareja; la edad promedio de los sujetos cuando cometieron el delito era de 34,02 años $(D S=9,98)$ y en el momento de la realización de este estudio de 39,77 años $(D S=10,63)$; por último, el 36,8\% de los sujetos tenía antecedentes penales, en su mayoría por delitos violentos.

\subsection{Instrumentos}

En consonancia con los objetivos de esta investigación, para evaluar los estadios de cambio en los que se encontraban los sujetos se empleó la Escala de Estadios de Cambio (SOCS). Este instrumento, que está disponible en la página web del Cancer Prevention Research Centre (de la Universidad de Rhode Island, EEUU, cuyo director es uno de sus diseñadores, James Prochaska), fue traducido al castellano, con posterior retrotraducción, a los efectos de este estudio (se incluye la versión castellana, aquí utilizada, como Apéndice). 
La escala SOCS es un cuestionario de autoinforme integrado por 32 ítems o afirmaciones sobre creencias y disposiciones que puede tener un individuo al respecto del problema de conducta que experimenta (en este caso acerca de sus delitos sexuales). Los participantes deben valorar, en una escala tipo Likert de 1 a 5 puntos, su grado de desacuerdo/acuerdo con lo planteado en cada ítem. Una puntuación cercana a 1 significa que el sujeto se siente en desacuerdo con el enunciado del ítem, mientras que una próxima a 5 indica que está muy de acuerdo con dicho enunciado.

Cada ítem de la escala se asocia a alguno de los cuatro estadios de cambio del modelo transteórico de Prochaska y DiClemente, constituyéndose así cuatro subescalas (precontemplación, contemplación, acción y mantenimiento). El sistema de corrección original de la escala SOCS, aquí usado, es categórico: a partir del sumatorio de puntuaciones de los ítems que componen cada subescala se obtiene una puntuación en cada uno de los cuatro estadios de cambio, lo que permite saber en cuál de ellos sobresale el individuo (Callagham y Taylor, 2006; DiClemente, Schlundt y Gemmell, 2004; Hemphill y Howell, 2000).

\subsection{Procedimiento}

Para la realización de este estudio se ha empleado un diseño de comparación de grupos. Como ya se ha señalado, la asignación de los sujetos a los grupos tomó como criterio el momento de tratamiento en el que éstos se encontraban (previo al tratamiento, durante el tratamiento, o tras haberlo finalizado).

El análisis de los datos se efectuó mediante el paquete estadístico SPSS 17. En primer lugar, se realizó un estudio de fiabilidad de la escala SOCS mediante el contraste de las correlaciones de Pearson y la obtención del estadístico alfa de Cronbach para las diferentes subescalas (correspondientes a los distintos estadios de cambio).

Para cada grupo se calcularon las puntuaciones medias y desviaciones típicas que presentaban sus correspondientes sujetos en cada uno de los estadios del modelo transteórico. Además, atendido el estadio de cambio en el que sobresalía cada sujeto, se obtuvieron, para cada grupo, los porcentajes de sujetos prevalentes en cada estadio. Las diferencias entre las puntuaciones medias de los grupos en los distintos estadios se exploraron mediante pruebas no-paramétricas. Se hizo así 
debido a que los datos no presentaban una distribución normal, requisito necesario para el uso de estadísticos paramétricos. Concretamente, se empleó la prueba Kruskal-Wallis para analizar las diferencias entre las medias de varios grupos independientes $\mathrm{y}$, finalmente, se aplicó la prueba $U$ de Mann-Whitney de comparación de medias entre dos grupos.

\section{RESULTADOS}

\subsection{Fiabilidad de la Escala de Estadios de Cambio (SOCS)}

La Tabla 1 muestra las correlaciones de Pearson entre las cuatro subescalas SOCS de estadios de cambio. Como puede verse, la subescala precontemplación correlaciona negativamente con la subescala contemplación, mientras que no muestra relación significativa con la subescalas acción y mantenimiento. Paralelamente, las subescalas contemplación, acción y mantenimiento muestran entre sí correlaciones positivas elevadas.

La consistencia interna de la Escala de Estadios de Cambio, explorada mediante el estadístico alfa de Cronbach, presenta valores altos para todas las subescalas, que oscilan entre $\alpha=0,72$ y 0,86 (Tabla 1 ).

Tabla 1. Análisis de fiabilidad de la Escala de Motivación al Cambio

\section{Correlación de Pearson}

Alfa de

Cronbach

Precontemplación Contemplación Acción Mantenimientc

\begin{tabular}{|c|c|c|c|c|c|}
\hline Precontemplación & - & & & & 0,72 \\
\hline Contemplación & $-0,192 *$ & - & & & 0,85 \\
\hline Acción & $-0,111$ & $0,852 * *$ & - & & 0,86 \\
\hline Mantenimiento & 0,000 & $0,683 * *$ & $0.641 * *$ & - & 0,80 \\
\hline
\end{tabular}

* Correlación significativa a $\mathrm{p}<0,05$

** Correlación significativa a $\mathrm{p}<0,01$ 


\subsection{Comparación entre los grupos}

En la Tabla 2 se presentan, para cada grupo, sus medias y desviaciones estándar en cada estadio de cambio, así como el porcentaje de sujetos que sobresale en él.

Los resultados del test Kruskal-Wallis indicaron diferencias de medias significativas entre los grupos en la subescala correspondiente al estadio precontemplación, $\mathrm{H}(2)=13.69, \mathrm{p}<0.01$, pero no en las restantes subescalas. La prueba $U$ de Mann Whitney reveló que tales diferencias en precontemplación se produjeron entre el grupo pre-tratamiento y el grupo tratamiento $(\mathrm{U}=762.00, z=$ $2.52, \mathrm{p}=0.01)$ y entre el grupo pre-tratamiento y el de seguimiento $(\mathrm{U}=316.00, z=$ $-3.34, \mathrm{p}<0.00)$.

En relación con el estadio de cambio en el que sobresale cada grupo, en precontemplación hay una mayor proporción de individuos del grupo pretratamiento (9\%). En el estadio contemplación prevalece el grupo tratamiento (con un 51,5\% de sus sujetos). En acción sobresale también el grupo pre-tratamiento (con el 56,7\%). Finalmente, en el estadio mantenimiento predomina el grupo seguimiento (con un 5,3\% de sus integrantes).

Tabla 2. Descripción de los resultados de la Escala de Motivación de Cambio

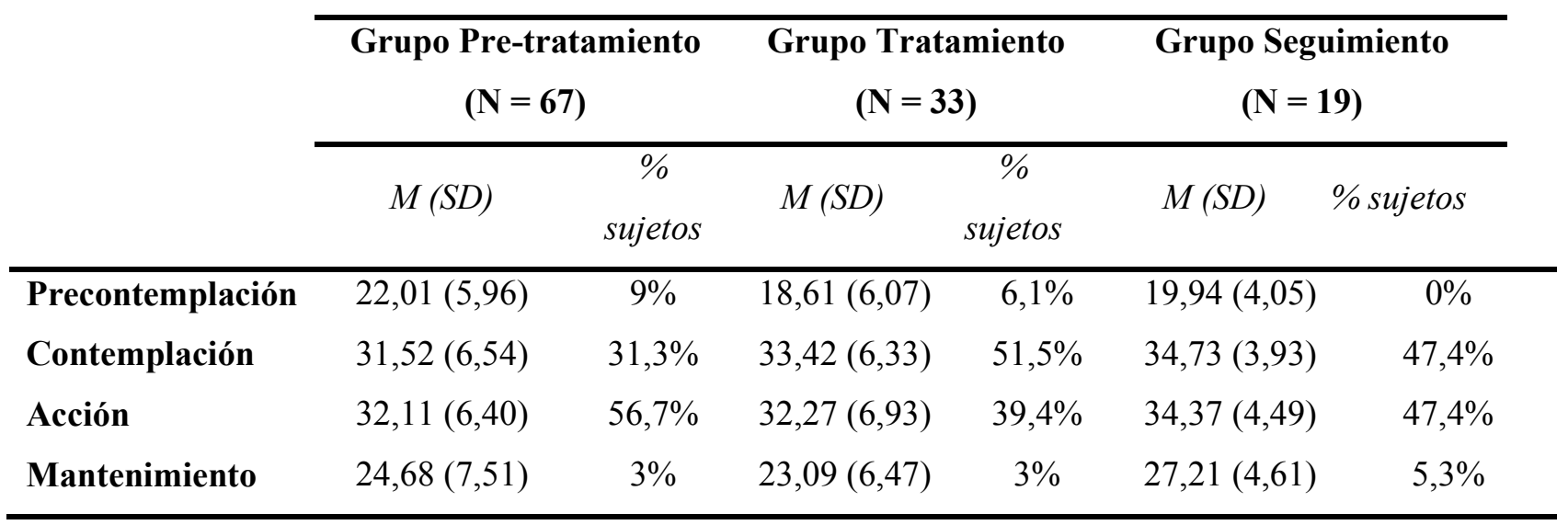

En la Figura 1 se representan, para cada grupo, los porcentajes acumulados de sujetos que prevalecen en cada estadio de cambio sucesivo. Los tres grupos de este estudio (pre-tratamiento, tratamiento y seguimiento) muestran un patrón acumulativo parecido. Con todo, los grupos tratamiento y seguimiento presentan menores proporciones de sujetos en precontemplación y tienden ligeramente a 
acumular mayor número de sujetos en estadios de cambio más avanzados, en contraste con el grupo pre-tratamiento.

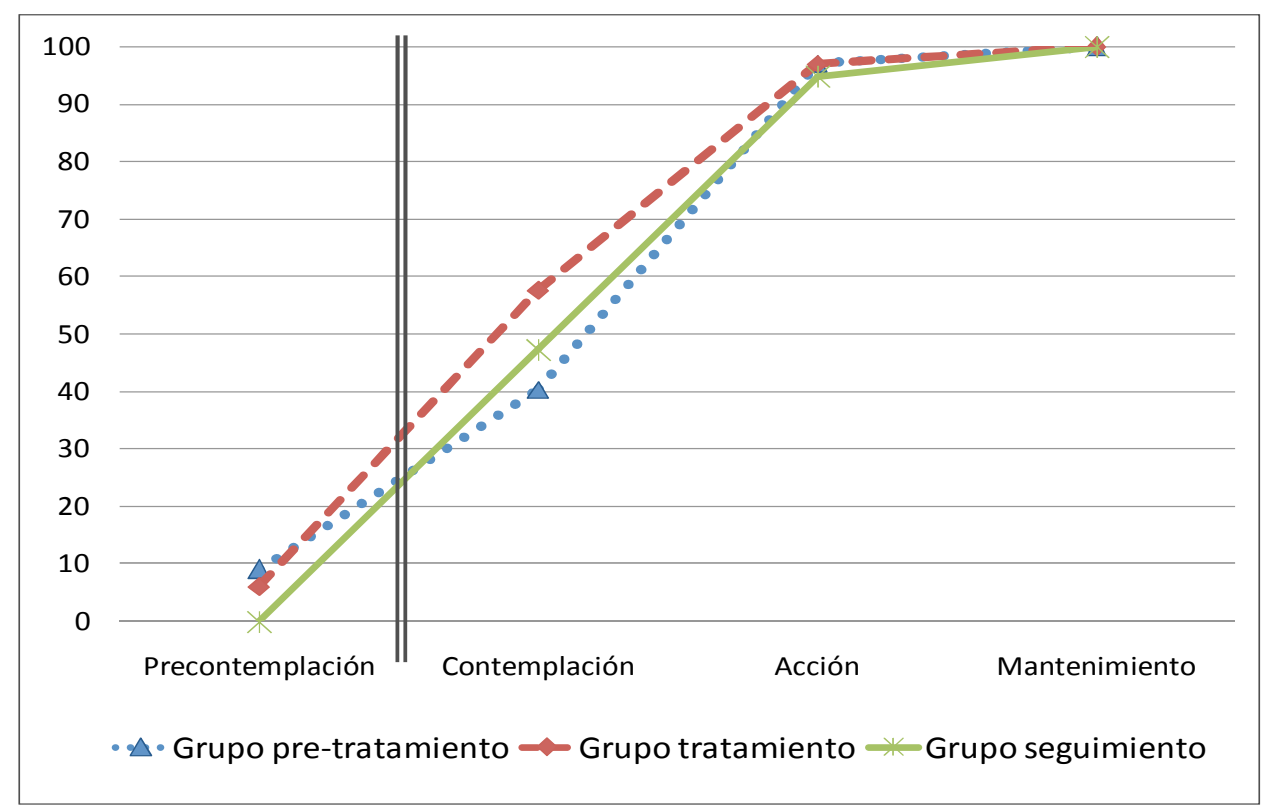

Figura 1. Porcentaje acumulado de sujetos prevalentes en cada estadio de cambio para cada grupo del estudio

\section{DISCUSIÓN Y CONCLUSIONES}

El objetivo principal de este estudio era explorar la utilidad del modelo transteórico de Prochaska y DiClemente (1982) en el marco del tratamiento y el cambio terapéutico de los delincuentes sexuales. Y en concreto conocer si existía relación entre tres momentos distintos del tratamiento (antes de recibirlo, en proceso, o habiéndolo finalizado) y el posicionamiento de los participantes en los estadios de cambio del modelo transteórico (precontemplación, contemplación, acción y mantenimiento).

Para evaluar a los sujetos y determinar en qué estadio de cambio se situaban fue utilizada la Escala de Estadios de Cambio (SOCS), traducida al castellano mediante retrotraducción. En ella los sujetos obtienen una puntuación para cada estadio de cambio del modelo. Las pruebas estadísticas realizadas avalan la fiabilidad de esta versión castellana de la escala. Las correlaciones entre sus diferentes subescalas, o estados de cambio, son consistentes con lo esperable. El estadio precontemplación (que indica falta de conciencia de problema) correlaciona 
inversamente con los estadios contemplación y acción, aunque no con seguimiento (estadios todos que representan, frente a precontemplación, la percepción de los individuos de que tienen en su vida un problema al que deben enfrentarse). Por su parte, estos tres últimos estadios, contemplación, acción y seguimiento, correlacionan positivamente entre sí, tal y como era de prever. Además, el estadístico alfa de Cronbach mostró valores altos para las diferentes subescalas o estadios de cambio, lo que permite concluir que la traducción al castellano de la escala SOCS, aquí presentada, cuenta con una buena consistencia interna. Debido a ello dicha traducción se ofrece como Apéndice de este artículo para facilitar que pueda ser utilizada por otros investigadores o profesionales interesados en este campo.

Como se mencionó, algunos autores han considerado que el tratamiento puede contribuir a incrementar la motivación de los delincuentes para el cambio terapéutico (Day, et al., 2008; Grubin y Thorton, 1994; Tierney y McCabe, 2004). En coherencia con ello, en este estudio se esperaba encontrar correspondencia entre el momento de tratamiento en que se hallasen los agresores sexuales y el estadio de cambio en el que se situaran. En concreto, había la expectativa de que los sujetos del grupo pre-tratamiento prevalecieran en estadios de cambio inferiores y los de los grupos tratamiento y seguimiento en estadios más avanzados. Los resultados obtenidos, mediante el test de Kruskal-Wallis y la prueba $U$ de Mann Whitney, corroboran la existencia de diferencias significativas entre los grupos en el estadio precontemplación, en el que el grupo pre-tratamiento puntúa significativamente más alto (es decir, es más inconsciente de su problema) que los grupos tratamiento y seguimiento, que puntúan más bajo (o sea, muestran mayor conciencia de tener un problema que resolver). En el resto de los estadios de cambio, en general se observan diferencias entre los grupos en las direcciones esperadas, aunque éstas no son estadísticamente significativas. Una limitación metodológica de este estudio es que los grupos evaluados están integrados por un número reducido de sujetos. Ello probablemente dificulta la obtención de resultados estadísticamente significativos, especialmente cuando las diferencias entre las puntuaciones de los grupos no son muy amplias.

Por otro lado, los análisis acerca de en qué estadios de cambio prevalecen los diversos grupos de estudio, así como la representación gráfica de porcentajes 
acumulados a lo largo de los sucesivos estadios, permiten llegar a una conclusión similar: el hecho de participar en un programa de tratamiento parece mejorar la motivación terapéutica y la disposición al cambio de conducta. Pese a todo, las diferencias entre los grupos en los distintos estadios de cambio no son amplias ni siempre se producen en el sentido esperado. Quizá el resultado más destacable sea la clara división que aparece entre el estadio precontemplación y los restantes estadios globalmente considerados. Mientras que en precontemplación destacan algo más los sujetos no tratados, los grupos tratamiento y seguimiento tienden a posicionarse en los tres estadios más altos, con la excepción de que también el grupo pre-tratamiento predomina en el estadio acción. Con la salvedad aludida, a medida que los sujetos avanzan en el tratamiento parece aumentar su disposición favorable a mejorar su conducta, mostrada en una tendencia a puntuar en estadios de cambio terapéutico superiores.

Según lo expuesto, la conclusión más positiva de esta investigación sería que la escala SOCS, que es sustentada por el modelo transteórico de Prochaska y DiClemente (1982), constituye una herramienta parcialmente útil para evaluar la disposición al cambio terapéutico de los agresores sexuales, lo que es consistente con estudios previos (Tierney y McCabe, 2004). Y, además, que esta escala podría ser utilizada como medida indirecta de eficacia terapéutica. No obstante, aunque la escala SOCS se limita a analizar los estadios de cambio, el cambio terapéutico es, según el propio modelo transteórico, un mecanismo más complejo que incluye también los procesos generadores de las mejoras terapéuticas y los niveles personales en que tales mejoras se producen (Prochaska, et al., 1992). Por otro lado, aunque este estudio presta atención al proceso interno de disposición para cambiar, que se considera imprescindible para la desistencia delictiva, no debe olvidarse que para que ésta se produzca también requiere que los individuos cuenten con nuevas oportunidades prosociales (Aresti, et al., 2010; Laub, et al., 1998). Por ello, un análisis más completo de los procesos de desistimiento del delito debería atender a ambos tipos de factores, y no sólo a los cambios internos objeto de esta investigación.

El resultado más débil quizá sea que la escala SOCS no muestra ser un instrumento muy sensible y preciso para evaluar la disposición al cambio y la eficacia terapéutica como resultado del tratamiento. Burrowes y Needs (2009) han 
puesto de relieve la dificultad de adaptar la escala de cambio al caso de los agresores encarcelados. El modelo de estadios de cambio fue originariamente diseñado como un análisis de situaciones terapéuticas de participación voluntaria en un tratamiento, en marcos comunitarios libres, en los que los participantes tienen la posibilidad de experimentar el tránsito natural por los diferentes estadios, y también los riesgos que se asocian a ellos (Norcross, et al., 2011). Por ejemplo, una adecuada plasmación del estadio mantenimiento presupondría que los sujetos en dicho estadio estuvieran expuestos a los riesgos naturales de su vida susceptibles de llevarles a una recaída, y que tal exposición, si es adecuadamente afrontada, acabara fortaleciendo su resistencia terapéutica. En cambio los agresores sexuales que aquí recibieron tratamiento se encuentran privados de libertad, lo que sin duda altera los procesos normales de motivación y cambio terapéuticos, tal y como se conceptúan desde la perspectiva del modelo transteórico. Todo ello puede hacer más difícil la adaptación de este modelo terapéutico y de su Escala de Estadios de Cambio al marco de situaciones de encarcelamiento.

Por último, el cambio en dirección a la desistencia delictiva no es un suceso repentino e irreversible sino, con toda probabilidad, un proceso gradual y reversible (Serin y Lloyd, 2009). Las personas cuando se plantean modificar ciertos comportamientos problemáticos e inician dicho cambio, no suelen hacerlo de un modo unidireccional, en el que no quepa ninguna vuelta atrás. Las recaídas parecen ser más la norma que la excepción. Además, según se vio, diferentes delincuentes sexuales podrían estar cambiando a distintos ritmos (Marshall y Pithers, 1994). Por ello, es razonable esperar que en la muestra de agresores sexuales aquí evaluados, individuos que todavía no participan en una intervención estén, pese a todo, considerando cambiar su conducta. Que sujetos que han iniciado tratamiento tengan todavía dudas sobre su deseo y disposición al cambio de comportamiento. Y que personas que ya han finalizado un programa terapéutico aún no se sientan completamente seguras, e incluso puedan volver atrás. Todos estos vaivenes y entrecruzamientos en dirección a la mejora de una conducta problemática y al retroceso en dicho logro, que probablemente constituyen la pauta general, podrían dificultar la obtención de resultados claros en una materia como el desistimiento de la delincuencia sexual. 


\section{REFERENCIAS}

Ajzen, I., \& Fishbein, M. (1980). Understanding attitudes and predicting social behaviour. New Jersey (NJ): Prentice Hall.

Alexander, P. C., \& Morris, E. (2008). Stages of change in batterers and their response to treatment. Violence and Victims, 23(4), 476-492.

Aresti, A., Eatough, V., \& Brooks-Gordon, B. (2010). Doing time after time: an interpretative phenomenological analysis of reformed exprisoners' experiences of self-change, identity and career opportunities. Psychology, Crime \& Law, 16(3), 169-190.

August, J. L., \& Flynn, A. (2007). Applying stage-wise treatment to a mixed-stage cooccurring disorders group. American Journal of Psychiatrc Rehabilitation, 10, 5363.

Bandura, A. (1977). Self-efficacy: Toward a unifying theory of behavioral change. Psychological Review, 84(2), 191-215.

Barbaree, H. (Ed.), \& Marshall, W. (Ed.). (2006). The juvenile sex offender (2nd ed.). New York, NY US: Guilford Press.

Barrett, M., Wilson, R. J., \& Long, C. (2003). Measuring Motivation to Change in Sexual Offenders From Institutional Intake to Community Treatment. Sexual Abuse. Journal of Research and Treatment, 15(4), 269-283.

Becker, M. H. (1974). The Health Belief Model and Personal Health Behavior. Health Education Monographs, 2(4).

Bowen, E., \& Gilchrist, E. (2004). Do court- and self-referred domestic violence offenders share the same characteristics? A preliminary comparison of motivation to change, locus of control and anger. Legal and Criminological Psychology, 9(2), 279-294.

Brown, S. (2005). Treating sex offenders: An introduction to sex offender treatment programmes. Devon (UK): Willan Publishing.

Burrowes, N., \& Needs, A. (2009). Time to contemplate change? A framework for assessing readiness to change with offenders. Aggression and Violent Behavior, 14(1), 39-49.

Callaghan, R. C., \& Taylor, L. (2006). Mismatch in the Transtheoretical Model? The American Journal on Addictions, 15(5). 
Casey, S., Day, A., \& Howells, K. (2005). The application of the transtheoretical model to offender populations: Some critical issues. Legal and Criminological Psychology, 10(2), 157-171.

Day, A., Bryan, J., Davey, L., \& Casey, S. (2006). The process of change in offender rehabilitation programmes. Psychology, Crime \& Law, 12(5), 473-487.

Day, A., Gerace, A., Wilson, C., \& Howells, K. (2008). Promoting forgiveness in violent offenders: A more positive approach to offender rehabilitation? Aggression and Violent Behavior, 13(3), 195-200.

DiClemente, C. C., \& Prochaska, J. O. (1982). Self-change and therapy change of smoking behavior: A comparison of processes of change in cessation and maintenance. Addictive Behaviors, 7(2), 133-142.

DiClemente, C. C., Garay, M., \& Gemmell, L. (2008). Motivational enhancement. En M. Galanter \& H. D. Kleber (Eds.), The American Psychiatric Publishing textbook of substance abuse treatment (4th ed.). (pp. 361-371). Arlington, (US): American Psychiatric Publishing, Inc.

DiClemente, C. C., Schlundt, D., \& Gemmell, L. (2004). Readiness and Stages of Change in Addiction Treatment. The American Journal On Addictions, 13(2), 103119.

Eckhardt, C. I., Babcock, J., \& Homack, S. (2004). Partner Assaultive Men and the Stages and Processes of Change. Journal of Family Violence, 19(2), 81-93.

Garland, R. J., \& Dougher, M. J. (1991). Motivational interventions in the treatment of sex offenders. En W. R. Miller \& M. S. Rollnick (Eds.), Motivational interviewing: preparing peolple to change addictive behaviour (pp. 303-313). New York (NY): Guildfrod Press.

Glanz, K., Rimer, B. K., \& Viswanath, K. (2008). Health behavior and health education: Theory, research, and practice (4th ed.). San Francisco, CA US: JosseyBass.

Grubin, D., \& Thornton, D. (1994). A national program for the assessment and treatment of sex offenders in the English prison system. Criminal Justice and Behavior, 21(1), 55-71.

Harkins, L., \& Beech, A. R. (2007). A review of the factors that can influence the effectiveness of sexual offender treatment: Risk, need, responsivity, and process issues. Aggression and Violent Behavior, 12(6), 615-627. 
Hemphill, J. F., \& Howell, A. J. (2000). Adolescent offenders and stages of change. Psychological Assessment, 12(4), 371-381.

Hollin, C. (Ed.), \& Palmer, E. (Ed.). (2006). Offending behaviour programmes: Development, application, and controversies. New York: John Wiley \& Sons Ltd.

Kear-Colwell, J., \& Pollock, P. (1997). Motivation or confrontation: Which approach to the child sex offender? Criminal Justice and Behavior, 24(1), 20-33.

Labrador, F. J., Echeburúa, E., \& Becona, E. (2000). Guía para la elección de tratamientos psicológicos efectivos. Madrid: Dykinson.

Laub, J. H., Nagin, D. S., \& Sampson, R. J. (1998). Trajectories of change in criminal offending: Good marriages and the desistance process. American Sociological Review, 63(2), 225-238.

LeBel, T. P., Burnett, R., Maruna, S., \& Bushway, S. (2008). The 'chicken and egg” of subjetive and social factors in desistance from crime. European Journal of Criminology, 5(2), 131-159.

Littrell, J. H., \& Girvin, H. (2002). Stages of change: A critique. Behavior Modification, 26(2), 223-273.

Marshall, L. E., \& Moulden, H. M. (2006). Preparatory programs for sexual offenders. En W. L. Marshall, Y. M. Fernandez, L. E. Marshall \& G. A. Serran (Eds.), Sexual offender treatment: Controversial issues. (pp. 199-210). New York, (US): John Wiley \& Sons Ltd.

Marshall, W. L., \& Pithers, W. D. (1994). A reconsideration of treatment outcome with sex offenders. Criminal Justice and Behavior, 21(1), 10-27.

McConnaughy, E. A., Prochaska, J. O., \& Velicer, W. F. (1983). Stages of change in psychotherapy: Measurement and sample profiles. Psychotherapy: Theory, Research \& Practice, 20(3), 368-375.

McGloin, J. M., Sullivan, C. J., Piquero, A. R., Blokland, A., \& Nieuwbeerta, P. (2011). Marriage and offending specialization: expanding the impact of turning point and the process of desistance. European Journal of Criminology, 8(5), 361-376.

McMurran, M., Theodosi, E., \& Sellen, J. (2006). Measuring engagement in therapy and motivation to change in adult prisoners: a brief report. Criminal Behavior and Mental Health, 16, 124-129. 
Miller, W. R. (1985). Motivation for treatment: a review with a special emphasis on alcohol. Psychological Bulletin, 98, 84-107.

Mullan, B., \& Westwood, J. (2010). The application of the theory of reasoned action to school nurses' behaviour. Journal of Research in Nursing, 15(3), 261-271.

Natan, M. B., Beyil, V., \& Neta, O. (2009). Nurses' perception of the quality of care they provide to hospitalized drug addicts: Testing the Theory of Reasoned Action. International Journal of Nursing Practice, 15(6), 566-573.

Nochajski, T. H., \& Stasiewicz, P. R. (2005). Assessing Stages of Change in DUI Offenders: A Comparison of Two Measures. Journal of Addictions Nursing, 16(12), 57-67.

Norcross, J. C., Krebs, P. M., \& Prochaska, J. O. (2011). Stages of change. Journal of Clinical Psychology, 67(2), 143-154.

Orbell, S., Lidierth, P., Henderson, C. J., Geeraert, N., Uller, C., Uskul, A. K., et al. (2009). Social-cognitive beliefs, alcohol, and tobacco use: A prospective community study of change following a ban on smoking in public places. Health Psychology, 28(6), 753-761.

Paulhus, D. L., \& Lim, D. T. K. (1994). Arousal and evaluative extremity in social judgments: A dynamic complexity model. European Journal of Social Psychology, 24(1), 89-99.

Paulhus, D. L., Trapnell, P. D., \& Chen, D. (1999). Birth order effects on personality and achievement within families. Psychological Science, 10(6), 482-488.

Pelissier, B. (2007). Treatment Retention in a Prison-based Residential Sex Offender Treatment Program. Sexual Abuse: A Journal of Research and Treatment, 19(4), 333-346.

Pereira de Oliveira, H., \& Malbieger, A. (2003). Assesment of motivation for treatment in alcohol dependent patients who sought treatment at a specialized medical service. Revista brasileña de psiquiatría, 25(1), 5-10.

Plotnikoff, R. C., Lippke, S., Trinh, L., Courneya, K. S., Birkett, N., \& Sigal, R. J. (2010). Protection motivation theory and the prediction of physical activity among adults with type 1 or type 2 diabetes in a large population sample. British Journal of Health Psychology, 15(3), 643-661. 
Polaschek, D. L., Anstiss, B., \& Wilson, M. (2010). The assessment of offendingrelated stage of change in offenders: Psychometric validation of the URICA with male prisoners. Psychology, Crime \& Law, 16(4), 305-325.

Prochaska, J. O., \& DiClemente, C. C. (1982). Transtheoretical therapy: Toward a more integrative model of change. Psychotherapy: Theory, Research \& Practice, 19(3), 276-288.

Prochaska, J. O., \& DiClemente, C. C. (2005). The Transtheoretical Approach. En J. C. Norcross \& M. R. Goldfried (Eds.), Handbook of psychotherapy integration (2nd ed.). (pp. 147-171). New York, NY US: Oxford University Press.

Prochaska, J. O., \& Norcross, J. C. (2001). Stages of change. Psychotherapy: Theory, Research, Practice, Training, 38(4), 443-448.

Prochaska, J. O., DiClemente, C. C., \& Norcross, J. C. (1992). In search of how people change: Applications to addictive behaviors. American Psychologist, 47(9), 11021114 .

Prochaska, J. O., Velicer, W. F., DiClemente, C. C., \& Fava, J. (1988). Measuring processes of change: Applications to the cessation of smoking. Journal of Consulting and Clinical Psychology, 56(4), 520-528.

Redondo, S. (2006). ¿Sirve el tratamiento para rehabilitar a los delincuentes sexuales? . Revista española de investigación criminológica, 6(4).

Redondo, S. (2008). Manual para el tratamiento psicológico de los delincuentes. Madrid: Pirámide.

Rogers, R. W. (1975). A protection motivation theory of fear appeals and attitude change. Journal of Psychology, 91, 93-114.

Rosen, C. S. (2000). Is the sequencing of change processes by stage consistent across health problems? A meta-analysis. Health Psychology, 19(6), 593-604.

Rosenstock, I. (1974). Historical Origins of the Health Belief Model. Health Education Monographs, 2(4).

Schunk, D. H., \& Pajares, F. (2009). Self-efficacy theory. En K. R. Wenzel \& A. Wigfield (Eds.), Handbook of motivation at school. (pp. 35-53). New York, (US): Routledge/Taylor \& Francis Group.

Scott, K. L., \& Wolfe, D. A. (2003). Readiness to change as a predictor of outcome in batterer treatment. Journal of Consulting and Clinical Psychology, 71(5), 879-889. 
Serin, R. C., \& Lloyd, C. D. (2009). Examining the process of offender change: The transition to crime desistance. Psychology, Crime \& Law, 15(4), 347-364.

Siegel, J. M. (1986). The Multidimensional Anger Inventory. Journal of Personality and Social Psychology, 51(1), 191-200.

Terry, K. J., \& Mitchell, E. W. (2001). Motivation and sex offender treatment efficacy: Leading a horse to water and making it drink? International Journal of Offender Therapy and Comparative Criminology, 45(6), 663-673.

Tierney, D. W., \& McCabe, M. (2002). Motivation for behavior change among sex offenders: A review of the literature. Clinical Psychology Review, 22(1), 113-129.

Tierney, D. W., \& McCabe, M. P. (2004). The assessment of motivation for behaviour change among sex offenders against children: An investigation of the utility of the Stages of Change Questionnaire. Journal of Sexual Aggression, 10(2), 237-249.

Tierney, D. W., \& McCabe, M. P. (2005). The Utility of the Trans-Theoretical Model of Behavior Change in the Treatment of Sex Offenders. Sexual Abuse: Journal of Research and Treatment, 17(2), 153-170.

Wells-Parker, E., Kenne, D. R., Spratke, K. L., \& Williams, M. T. (2000). Self-efficacy and motivation for controlling drinking and drinking/driving: an investigation of changes across a driving under the influence (DUI) intervention program ad of recidivism prediction. Addictive Behaviours, 25(2), 229-238.

Williamson, P., Day, A., Howells, K., Bubner, S., \& Jauncey, S. (2003). Assessing offender readiness to change problems with anger. Psychology, Crime \& Law, 9(4), 295-307. 


\section{APÉNDICE}

Versión en castellano de la Escala de Estadios de Cambio-SOCS de Prochaska y DiClemente

PUNTUACIONES DEL SUJETO EN CADA ESTADIO DE CAMBIO

\begin{tabular}{|l|l|l|l||}
\hline $\mathrm{P}$ & $\mathrm{C}$ & $\mathrm{A}$ & $\mathrm{M}$ \\
\hline & & & \\
\hline
\end{tabular}

Lea atentamente las siguientes afirmaciones e indicque su grado de acuerdo con cada una de ellas, en base a la escala de cinco puntos que se especifica a continuación. Bases sus respuestas en cómo se siente y se comporta usted en la actualidad.

$1=$ Completamente en desacuerdo

$2=$ En desacuerdo

$3=$ No tengo opinión

$4=$ De acuerdo

$5=$ Completamente de acuerdo

\begin{tabular}{|c|c|c|c|c|c|}
\hline 1. Que yo sepa, no tengo ningún problema que necesite cambiar & 1 & 2 & 3 & 4 & 5 \\
\hline 2. Creo que puedo estar preparado para mejorar algo de mí mismo & 1 & 2 & 3 & 4 & 5 \\
\hline $\begin{array}{l}\text { 3. Estoy empezando a poner remedio a los problemas que me han } \\
\text { estado preocupando }\end{array}$ & 1 & 2 & 3 & 4 & 5 \\
\hline 4. Puede valer la pena que me esfuerce para solucionar mi problema & 1 & 2 & 3 & 4 & 5 \\
\hline $\begin{array}{l}\text { 5. No soy una persona que tenga problemas. No tiene ningún sentido } \\
\text { el que yo esté aquí }\end{array}$ & 1 & 2 & 3 & 4 & 5 \\
\hline $\begin{array}{l}\text { 6. Me preocupa recaer en un problema que ya he solucionado, por eso } \\
\text { estoy aquí para pedir ayuda }\end{array}$ & 1 & 2 & 3 & 4 & 5 \\
\hline 7. Por fin estoy haciendo algo para resolver mis problemas. & 1 & 2 & 3 & 4 & 5 \\
\hline 8. He estado pensando que tal vez quiera cambiar algo de Mí mismo & 1 & 2 & 3 & 4 & 5 \\
\hline $\begin{array}{l}\text { 9. He conseguido solucionar mi problema, pero no estoy seguro de } \\
\text { poder mantener el esfuerzo por mí mismo }\end{array}$ & 1 & 2 & 3 & 4 & 5 \\
\hline $\begin{array}{l}\text { 10. A veces mi problema es difícil, pero continúo esforzándome para } \\
\text { solucionarlo }\end{array}$ & 1 & 2 & 3 & 4 & 5 \\
\hline $\begin{array}{l}\text { 11. Estar aquí es casi una pérdida de tiempo ya que el problema no } \\
\text { tiene que ver conmigo }\end{array}$ & 1 & 2 & 3 & 4 & 5 \\
\hline 12. Espero que aquí me ayuden a comprenderme mejor a mí mismo & 1 & 2 & 3 & 4 & 5 \\
\hline $\begin{array}{l}\text { 13. Supongo que tengo defectos, pero no hay nada que realmente yo } \\
\text { necesite cambiar }\end{array}$ & 1 & 2 & 3 & 4 & 5 \\
\hline 14. Estoy haciendo un gran esfuerzo para cambiar & 1 & 2 & 3 & 4 & 5 \\
\hline 15. Tengo un problema y creo que debería intentar solucionarlo & 1 & 2 & 3 & 4 & 5 \\
\hline
\end{tabular}




\begin{tabular}{|c|c|c|c|c|c|}
\hline $\begin{array}{l}\text { 16. No me siento capaz de mantener los cambios que he logrado, y } \\
\text { estoy aquí para prevenir una recaída en mi problema }\end{array}$ & 1 & 2 & 3 & 4 & 5 \\
\hline $\begin{array}{l}\text { 17. Aunque no siempre consigo el mejor resultado, al menos lo estoy } \\
\text { intentando }\end{array}$ & 1 & 2 & 3 & 4 & 5 \\
\hline $\begin{array}{l}\text { 18. Pensaba que una vez que hubiera resuelto mi problema me sentiría } \\
\text { liberado, pero a veces aún me encuentro luchando con él }\end{array}$ & 1 & 2 & 3 & 4 & 5 \\
\hline 19. Desearía tener más ideas sobre cómo resolver mi problema & 1 & 2 & 3 & 4 & 5 \\
\hline $\begin{array}{l}\text { 20. He empezado a hacer frente a mis problemas, pero me gustaría que } \\
\text { me ayudaran }\end{array}$ & 1 & 2 & 3 & 4 & 5 \\
\hline 21. Quizás en este lugar me puedan ayudar & 1 & 2 & 3 & 4 & 5 \\
\hline $\begin{array}{l}\text { 22. Puede que ahora necesite un empuje para ayudarme a mantener los } \\
\text { cambios que he conseguido }\end{array}$ & 1 & 2 & 3 & 4 & 5 \\
\hline 23. Puede que yo sea parte del problema, pero no todo el problema & 1 & 2 & 3 & 4 & 5 \\
\hline 24. Espero que alguien de aquí me pueda dar un buen consejo & 1 & 2 & 3 & 4 & 5 \\
\hline $\begin{array}{l}\text { 25. Cualquiera puede hablar sobre cambiar; pero yo estoy haciendo } \\
\text { algo al respecto }\end{array}$ & 1 & 2 & 3 & 4 & 5 \\
\hline $\begin{array}{l}\text { 26. Toda esta charla psicológica es aburrida. ¿Por qué no puede la } \\
\text { gente simplemente olvidar sus problemas? }\end{array}$ & 1 & 2 & 3 & 4 & 5 \\
\hline 27. Estoy aquí para prevenir la posibilidad de recaer en mi problema. & 1 & 2 & 3 & 4 & 5 \\
\hline $\begin{array}{l}\text { 28. Es frustrante, pero siento que podría reaparecer un problema que } \\
\text { creía tener ya resuelto }\end{array}$ & 1 & 2 & 3 & 4 & 5 \\
\hline $\begin{array}{l}\text { 29. Tengo tantas preocupaciones como cualquier otra persona. ¿Por } \\
\text { qué perder el tiempo pensando en ellas? }\end{array}$ & 1 & 2 & 3 & 4 & 5 \\
\hline 30. Estoy trabajando activamente para solucionar mi problema & 1 & 2 & 3 & 4 & 5 \\
\hline 31. Preferiría convivir con mis defectos antes que intentar cambiarlos & 1 & 2 & 3 & 4 & 5 \\
\hline $\begin{array}{l}\text { 32. Después de todo lo que he hecho para intentar solucionar mi } \\
\text { problema, reaparece una y otra vez }\end{array}$ & 1 & 2 & 3 & 4 & 5 \\
\hline
\end{tabular}

\section{Valoraciones globales complementarias a la escala SOCS}

Mirándome a mí mismo con absoluta sinceridad, considero que el riesgo existente de volver a caer en mi problema es, en una escala de 0 a 10 puntos, de ...... PUNTOS, siendo 0 un riesgo nulo y 10 un riesgo máximo.

\section{CONTESTE A LO SIGUIENTE, SÓLO SI HA PARTICIPADO EN UN} PROGRAMA DE TRATAMIENTO

Indique, de 1 a 7 puntos, en qué grado considera haber mejorado globalmente como resultado del tratamiento recibido: 
SANTIAGO REDONDO ILLESCAS es Profesor titular de Criminología y Psicología de la Universidad de Barcelona. También ha sido profesor invitado en diversas universidades españolas y de otros países para impartir cursos y conferencias sobre temáticas como la criminalidad en las sociedades actuales, la delincuencia juvenil, el tratamiento y la rehabilitación de los agresores y delincuentes, las explicaciones científicas del delito, las agresiones sexuales y el maltrato de pareja. Actualmente dirige diversas investigaciones en torno al tratamiento de los agresores juveniles y de los delincuentes sexuales. También es investigador invitado en varios proyectos internacionales sobre la transición desde la delincuencia juvenil a la delincuencia adulta, y sobre tratamientos eficaces con delincuentes. Es autor de una teoría integradora sobre la delincuencia denominada Modelo del Triple Riesgo Delictivo (TRD). Ha publicado numerosos artículos científicos, capítulos de libros y libros. Sus libros más recientes son los titulados Principios de Criminología (Valencia, 2006), Manual para el tratamiento psicológico de los delincuentes (Madrid, 2008), In-tolerancia cero: un mundo con menos normas, controles y sanciones también sería posible (Barcelona, 2009), y ¿Por qué víctima es femenino y agresor masculino? La violencia de pareja y las agresiones sexuales (Madrid, 2010).

ANA MARTíneZ CATENA es licencia en Psicología y Criminología por la Universidad de Barcelona, y es Máster en Psicología Forense y Criminal, y en Investigación en Personalidad y Comportamiento. En la actualidad realiza su tesis doctoral en materia de tratamiento de la delincuencia y motivación para el cambio de conducta. Sus intereses de investigación giran en torno a la delincuencia juvenil y las carreras delictivas, especialmente los factores críticos implicados en la transición de comportamiento delictivo juvenil al adulto y en la desistencia del delito. Colabora con el profesor Santiago Redondo Illescas, lo que le ha permitido participar en diversas investigaciones relacionadas con la predicción, tratamiento y prevención del comportamiento violento.

Agradecimientos: Este trabajo se realizó en el marco del proyecto de investigación SEJ2005-09170-C04-01, financiado por el Ministerio de Educación y Ciencia de España, y el proyecto PSI2009-13265, financiado por el Ministerio de Ciencia e Innovación de España. Los autores quieren agradecer la cooperación del Departamento de Justicia de Cataluña para la realización de esta investigación, así como el apoyo y ayuda de Juan Carles Navarro, Meritxell Pérez y Meritxell Navarro. 